\title{
Charged solitons in branched conducting polymers
}

\author{
D. Babajanov ${ }^{a}$, H. Matyoqubov ${ }^{b}$, and D. Matrasulov ${ }^{a}$ \\ a Turin Polytechnic University in Tashkent, 17 Niyazov Str., 100095, Tashkent, Uzbekistan \\ ${ }^{b}$ Urgench State University, 14 H. Olimjon Str., 220100, Urgench, Uzbekistan
}

\begin{abstract}
We consider dynamics of charged solitons in branched conducting polymers. An effective model based on the sine-Gordon equation on metric graphs is used for computing the charge transport and scattering of charge carriers at the polymer branching points. Condition for the ballistic charge carrier transportis revealed.
\end{abstract}

\section{INTRODUCTION}

Conducting polymers are the basic materials for organic electronics, including polymer based photovoltaics. Effective functionalization of such materials requires understanding the mechanisms for charge carrier transport and their optimal control. Considerable progress has been made in the study of charge transport mechanisms in conducing polymers during past three decades [1][7]. In particular, several mechanisms for charge transport, such as exciton, polaron and soliton based ones have been proposed. Excitons, which are the quasiparticle consisting of bound electron-hole pair, can be induced e.g., in conjugated polymers, due to the photon impact. Trapping and travelling of charge by kink solitons in conducting polymers provide another mechanism for charge transport. When neutral and charged solitons appear in bound state, they can form charge carriers, which are called polarons. Each mechanism may play key role depending on the type of a device and functionalization method. Therefore effective utilization of these mechanisms in organic electronics requires developing different realistic models for charge carrier dynamics. Most of the conducting polymers are typically composed of linear conjugated systems, which can be observed, e.g., in the study of polyacetylene, polypyrrole, polyaniline, polythiophene, and other conjugated polymers. However, conducting polymers having branched architecture attracted much attention recently. These
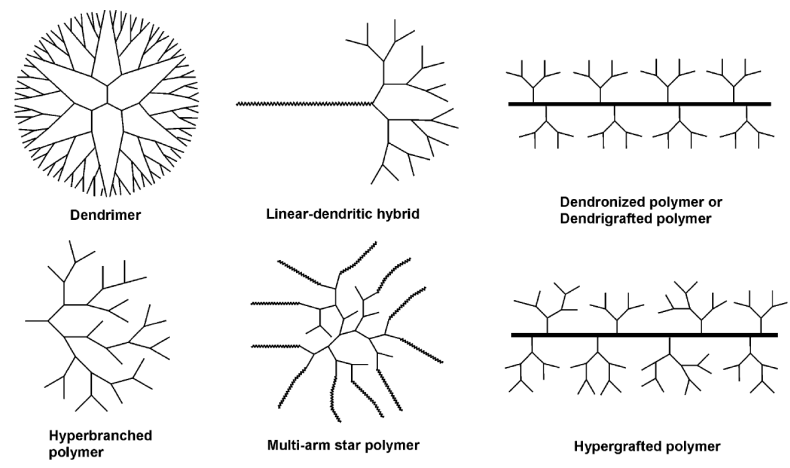

FIG. 1: Schematic description of dendritic polymers (from the Ref. 14]).

are kind of polymers in which linear chain splits into the two or more branches starting from some point, which is called branching point, or node, or vertex. The structure of a branching can have different architecture, e.g. can be in form of star, tree, ring, etc. These latter implies the rule for branching and called branching topology. When the topology of a polymer is very complicated, it is called hyperbranched polymer. Branched polymers differ from their linear counterparts in several important aspects. Such polymer forms a more compact coil than a linear polymer with the same molecular weight. Also, depending on the topology of branching, electronic and elasticity properties can be completely different than those of linear polymers. A number of papers on the synthesis of different branched polymers and study of their optical, electronic and mechanical properties have been published in the literature during past two decades (see, e.g., [8]- 23]). A review of the chemistry and physics of hyperbranched polymers is presented in [11]. In [15] a synthesis strategy for a hyperbranched sulfonated polydiphenylamine was developed and electronic properties have been studied. Synthesis and light-emitting applications of several conjugated polymers have been reviewed in [16]. In 17] the synthesis of dendrimer-like starbranched polymers is reported. Scalable and versatile synthesis of multifunctional polyaniline with branched structure with excellent electronic conductivity and electrochemical properties was reported in [19]. A review of the synthesis, properties, and functionality of dendrimers, hyperbranched polymer, and star polymers with emphasis on functional aspects can be found in 15]. Synthesis and optical properties of organosiliconeoligothiophene branched polymers was reported in [20]. Fabrication and electronic properties of functionalized branched EDOT-terthiophene copolymer films was considered in 22].

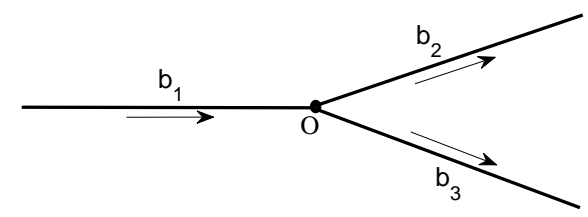

FIG. 2: Basic star graph. 
a)

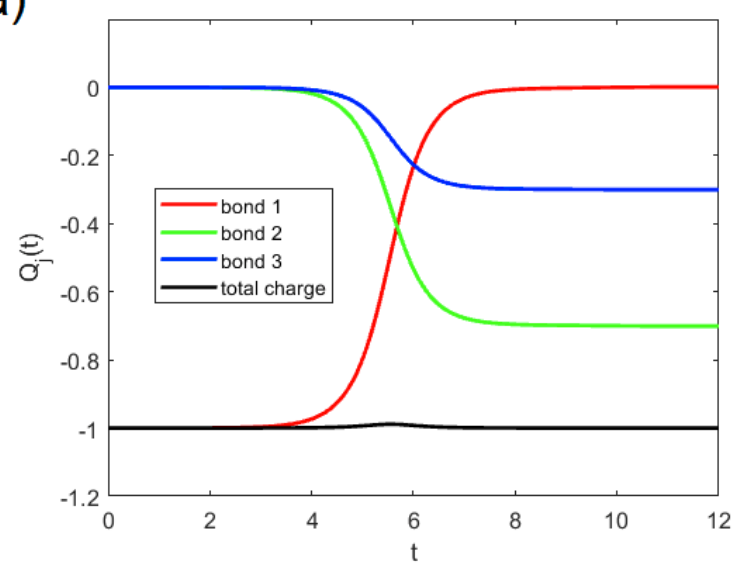

b)

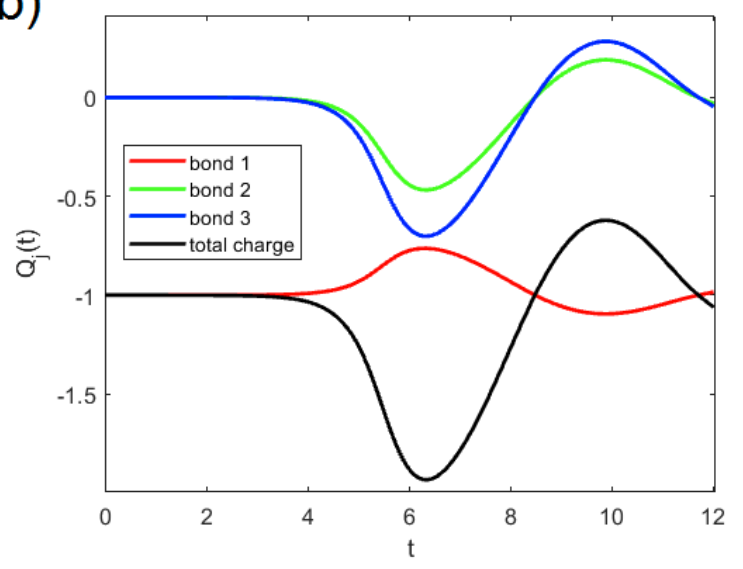

FIG. 3: Time dependence of charge on each branch of the star branched polymer: (a) when the sum rule (2.6) is fulfilled $\left(a_{1}=1, a_{2}=0.7, a_{3}=0.3\right.$. $)$ and (b) broken $\left(a_{1}=1, a_{2}=\right.$ $2, a_{3}=3$.)

Some physical properties of conducting polymer networks are discussed in [24, 25]. Despite the considerable progress made in the synthesis and study of branched conducting polymers, the problem of the charge carrier dynamics in such structures is still remaining as less studied topic.

In this paper we consider dynamics of charged solitons in branched conducting polymers. Solitons as charge carriers in linear conducting polymers have been extensively studied earlier in the literature [26] - 31]. Here we propose a model based on the sine-Gordon equation on metric graphs to describe charged soliton transport in branched conducting polymers. The model considers the branched polymer as a macroscopic network, where the soliton dynamics is described in terms of a nonlinear wave equation. Using the exact and numerical solutions of the problem, we study the problem of charge transport, scattering and transmission of charged solitons through the branching point. Within the proposed model, we reveal the conditions for ballistic and diffusive transport of charged solitons in branched conducting polymers.

We note that particle [37]- [41] and soliton dynamics in

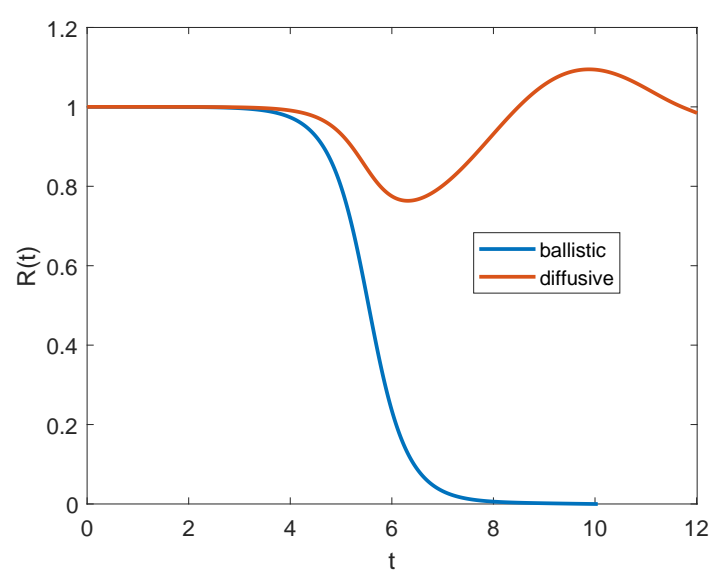

FIG. 4: Time-dependence of the branching point reflection coefficient (defined as $R(t)=Q_{1}(t) / Q_{1}(t=0)$ ) for the charged soliton in star branched polymer in cases of ballistic and diffusive transport.

networks has been the topic for extensive research during past decade (see, e.g., Refs. [43] -[52]). Modeling such structures in terms of metric graphs provides powerful tool for effective description of the wave dynamics in branched structures appearing in different areas of physics. The graph itself is determined as a set of branches which are connected to each other at the vertices (branching points) according to some rule. This rule is called the topology of a graph. When branches of a graph are assigned length it is called matric graph. Topology of a graph is given in terms of the adjacency matrix which is defined, e.g., in [37 41].

Simplest graph topology is called basic star graph, which presents simple Y-junction with three branches. Advantage of modeling branched structures by metric graphs is the fact that it allows to describe the structure as one-dimensional, or quasi-one dimensional system. Here we consider star, loop and tree shaped polymers. This paper is organized as follows. In the next section we give formulation of the problem together with the description of the model for star branched polymers. Section III extends to the model for other branching topologies, such as loop- and tree-shaped polymers. Finally, section IV presents some concluding remarks.

\section{DYNAMICS OF SINE-GORDON SOLITONS IN BRANCHED POLYMERS: MODELING FOR STAR-SHAPED TOPOLOGY}

Most of the branched polymers synthesized so far, have star or dendritic hyperbranched structures, although some other architectures including those having fractal structure are available. Some of the hyperbranched topologies are presented in Fig. [1 There are several models for charged soliton dynamics in conducting polymers, such as Su-Schrieffer-Heeger [33], Pariser- 

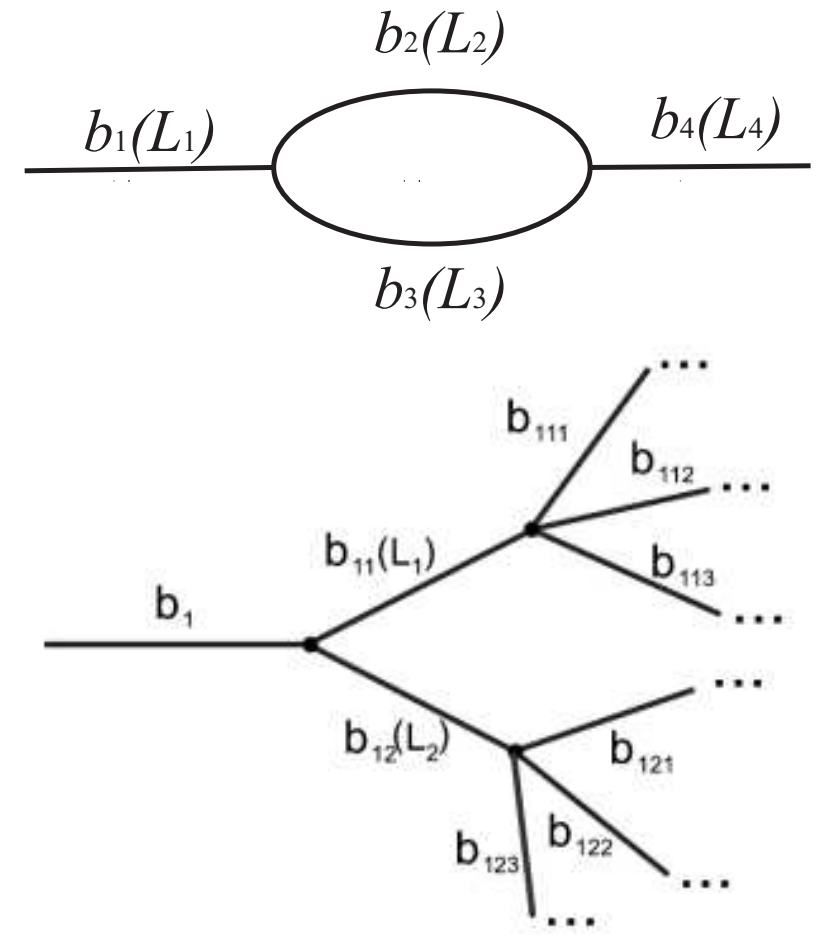

FIG. 5: Loop (upper) and tree (lower) graphs.

Parr-Pople [35], time-dependent Hartree-Fock 36] models. Here we develop a model that considers charged soliton as sine-Gordon kink. Within such approach, charged soliton dynamics in branched polymers can be described in terms of the sine-Gordon equation on metric graphs. Let us first consider simplest case, a star branched polymer having the form of Y-junction. We assume that the branches are very long compared to the thickness of the polymer chain. Then such polymer can be considered as a basic star graph with semi-infinite branches connected at the point $O$ called vertex, or branching point of the graph (see Fig 2). The coordinates of soliton in such structure are defined as $x_{1} \in(-\infty, 0]$ and $x_{2,3} \in[0, \infty)$, where 0 corresponds to the branching point. Dynamics of charged soliton in such branched polymer can be described in terms of the sine-Gordon equation on metric graph which can be written on each branch as [51]

$$
\psi_{k t t}-a_{k}^{2} \psi_{k x x}+\beta_{k} \sin \psi_{k}=0 .
$$

To solve this equation, one needs to impose the boundary conditions at the branching point (vertex) of the graph and determine the asymptotic of the wave function at the branch ends. To formulate vertex boundary conditions (VBC) one can use the continuity of wave function 51]

$$
\psi_{1}(0, t)=\psi_{2}(0, t)=\psi_{3}(0, t)
$$

and fundamental conservation laws such as energy, charge and momentum conservations. The asymptotic conditions at infinities can be written as $\partial_{x} \psi_{1}\left(x_{1}, t\right), \partial_{t} \psi_{1}\left(x_{1}, t\right) \rightarrow 0$ and $\psi_{1}\left(x_{1}, t\right) \rightarrow 2 \pi n_{1}$ as $x_{1} \rightarrow$
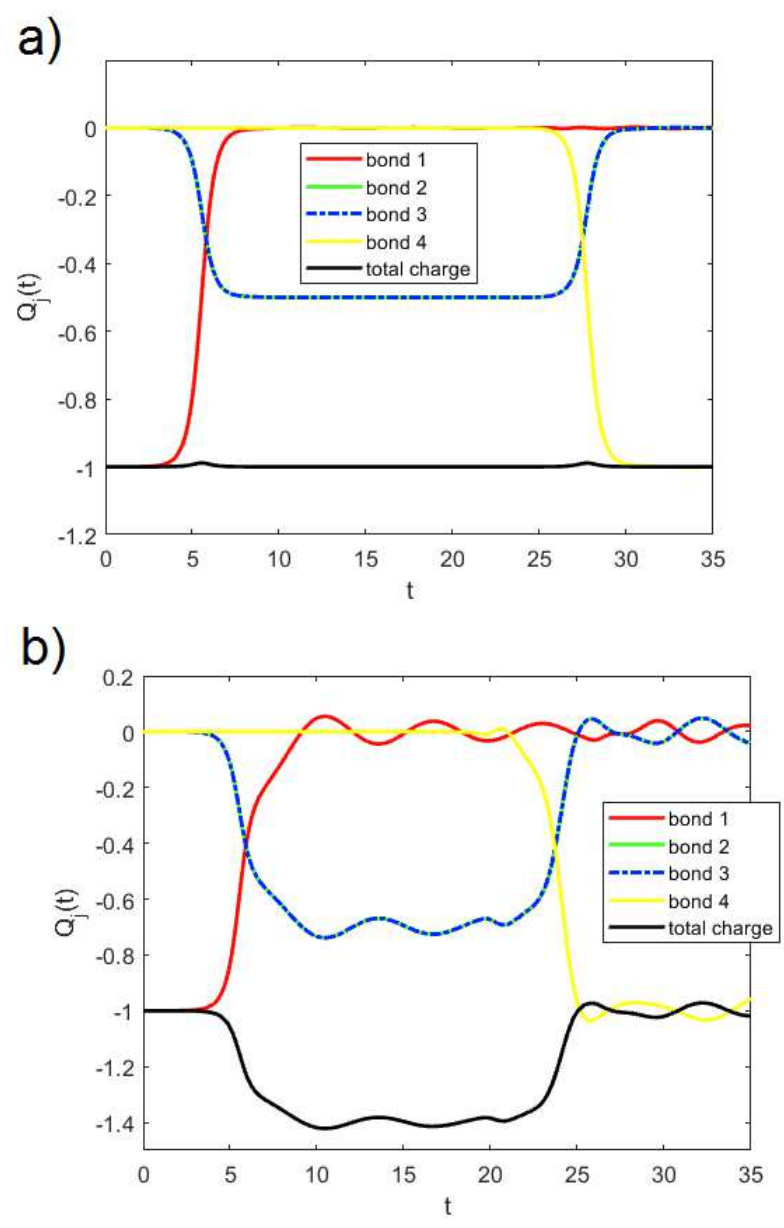

FIG. 6: Time dependence of charge on each branch of the loop branched polymer when (a) the sum rule is fulfilled $\left(a_{1}=\right.$ $1, a_{2}=0.5, a_{3}=0.5, a_{4}=1$.) and (b) broken $\left(a_{1}=1, a_{2}=\right.$ $\left.0.7, a_{3}=0.7, a_{4}=1\right)$.

$-\infty$, and $\partial_{x} \psi_{k}\left(x_{k}, t\right), \partial_{t} \psi_{k}\left(x_{k}, t\right) \rightarrow 0$ and $\psi_{k}\left(x_{k}, t\right) \rightarrow$ $2 \pi n_{k}$ as $x_{k} \rightarrow \infty, k=2,3$, for some integer $n_{k}, k=$ $1,2,3$.

For the star graph in Fig 2, the energy and topological charge are defined as (respectively) [51]

$E(t)=\sum_{k=1}^{3} \frac{1}{\beta_{k}} \int_{B_{k}}\left[\frac{1}{2}\left(\psi_{k t}^{2}+a_{k}^{2} \psi_{k x}^{2}\right)+\beta_{k}\left(1-\cos \psi_{k}\right)\right] d x$,

and

$$
2 \pi Q=\frac{a_{1}}{\sqrt{\beta_{1}}} \int_{-\infty}^{0} \psi_{1 x} d x+\sum_{k=2}^{3} \frac{a_{k}}{\sqrt{\beta_{k}}} \int_{0}^{+\infty} \psi_{k x} d x,
$$

where $B_{1}=(-\infty, 0), B_{2,3}=(0,+\infty)$. From the conservation laws given by

$$
\frac{d E}{d t}=0, \quad \frac{d Q}{d t}=0,
$$

we have the boundary conditions at the branching point: 


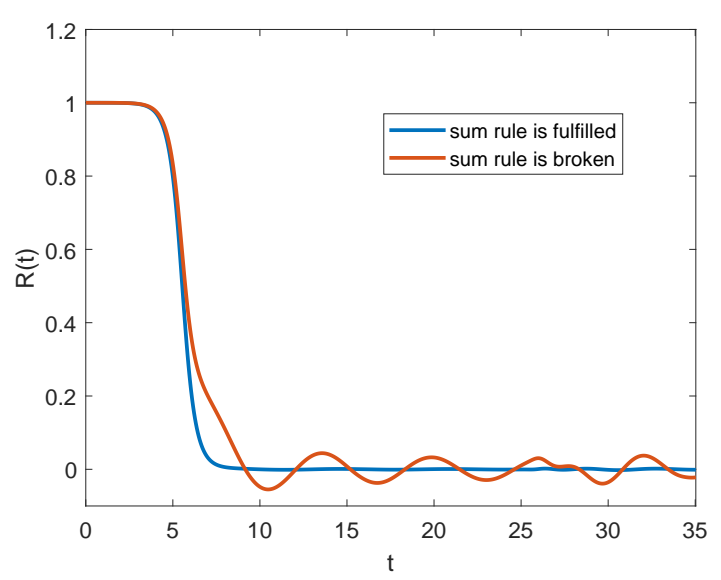

FIG. 7: Time-dependence of the branching point reflection coefficient (defined as $R(t)=Q_{1}(t) / Q_{1}(t=0)$ ) for loop branched polymer in ballistic and diffusive regimes.

$$
\left.\frac{a_{1}^{2}}{\beta_{1}} \psi_{1 x}\right|_{x_{1}=0}=\left.\frac{a_{2}^{2}}{\beta_{2}} \psi_{2 x}\right|_{x_{2}=0}+\left.\frac{a_{3}^{2}}{\beta_{3}} \psi_{3 x}\right|_{x_{3}=0} .
$$

It was shown in [51] that exact soliton (kink)solutions of Eq.(2.1) fulfilling the vertex boundary conditions given by Eqs.2.2) and (2.5) can be obtained, provided the following sum rule holds true:

$$
\frac{a_{1}}{\sqrt{\beta_{1}}}=\frac{a_{2}}{\sqrt{\beta_{2}}}+\frac{a_{3}}{\sqrt{\beta_{3}}} .
$$

Then the solution can be written as

$$
\psi(x, t)=v\left(\frac{\sqrt{\beta_{k}}}{a_{k}} x, \sqrt{\beta_{k}} t\right),
$$

where

$$
v(x, t)=4 \arctan \left[\exp \left( \pm \frac{x-x_{0}-\nu t}{\sqrt{1-\nu^{2}}}\right)\right],
$$

with $|\nu|<1$ being the velocity of the kink.

Thus dynamics of the charged solitons in branched conducting polymers is described in terms of the problem given by Eqs.(2.1), (2.2) and (2.5). In other words, within our model, solution of sine-Gordon equation on such graph describes the motion of charged kink solitons in branched conducting polymers. Detailed mathematical treatment of this problem was given recently in the Ref. [51]. Here we apply these results to the problem of charged soliton transport in branched conducting polymers by considering different branching topologies. Having the solution of the problem given by Eqs. (2.1), (2.2) and (2.5), one can compute different characteristics of the charge transport, such as time-evolution of the topological charge, reflection and transmission of charge carrying solitons at the polymer branching point. In the following for numerical computations we assume that $\beta_{1}=\beta_{2}=\beta_{2}=1$. Fig. 3 a presents plots of the charge as a function of time on each branch of the a)

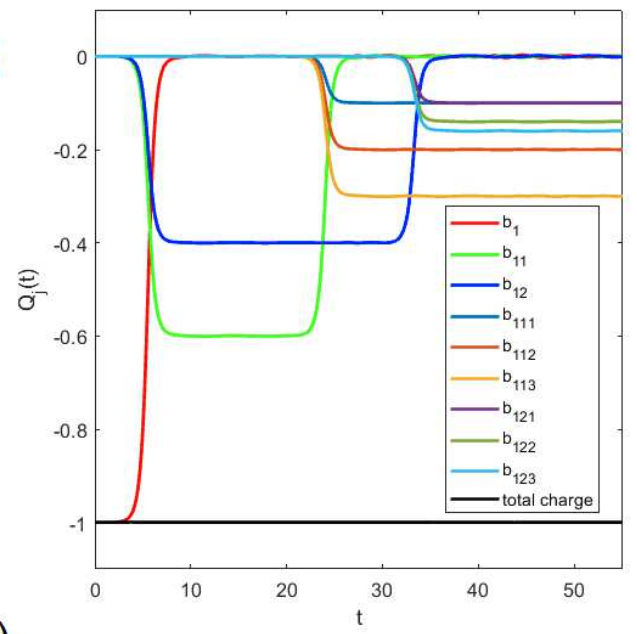

b)

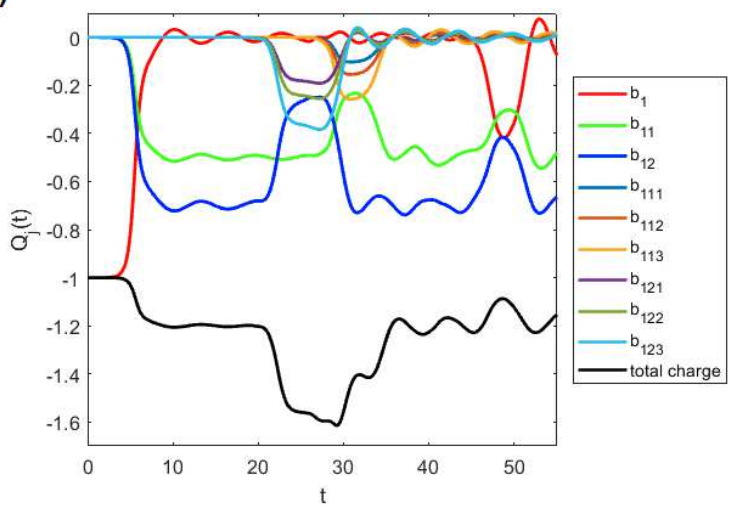

FIG. 8: Time dependence of charge on each branch of the tree branched polymer when (a) the sum rule is fulfilled $\left(a_{1}=1, a_{11}=0.6, a_{12}=0.4, a_{111}=0.1, a_{112}=0.2, a_{113}=\right.$ $\left.0.3, a_{121}=0.1, a_{122}=0.14, a_{123}=0.16\right)$ and (b) broken $\left(a_{1}=1, a_{11}=0.5, a_{12}=0.7, a_{111}=0.2, a_{112}=0.3, a_{113}=\right.$ $\left.0.5, a_{121}=0.3, a_{122}=0.4, a_{123}=0.6\right)$.

star-branched-polymer for the regime, when the sum rule given by Eq.(2.6) is fulfilled, i.e. the case when energy and charge conservations hold true. Conservation of topological charge can be clearly seen from this plot. Fig. [3 presents similar plots for the case, when sum rule is broken. It is clear that the charge conservation is not fulfilled in this regime. In both cases it is assumed that charge is generated on the first branch at the initial time $(t=0)$. In Fig. 4 reflection coefficient of charged solitons from the (star-shaped) polymer branching points are plotted for the regimes, when the sum rule given by Eq.(2.6) is fulfilled and broken. As it can be seen from these plots, there is no reflection of solitons at the branching point, when the sum rule is fulfilled. However, for the case, when the sum rule is broken, one can observe reflection and backward motion of the charge solitons at the branching point. Such an effect can be effectively used for tuning of charge transport in branched conducting polymers and organic electronic devices fabricated on their basis. 


\section{OTHER BRANCHING TOPOLOGIES}

The above approach can be extended for modeling of charged soliton dynamics in other branched polymers having more complicated branching topologies. Consider first the loop branched polymer which consists of two semi-infinite branches connected by two or more branches having finite lengths $L_{1}$ and $L-2$. Such polymer can be considered as a loop graph presented in Fig.(50)). Under the constraints given as [51]

$$
a_{1}=a_{1}+a_{2}=a_{4}
$$

for the coefficients, and $L_{k}=a_{k} L(k=1,2,3,4)$ with a constant $L$, one can write exact soliton solutions of sineGordon equation on such structure. In other words, the kink solution given by Eq.(2.8) is the solution of Eq.(2.1) provided coefficients $a_{j}$ fulfill the sum rule (3.1) [51]. When the sum rule is broken, one can solve the problem only numerically. Having found such solutions, one can compute different characteristics of the charge transport. Fig. (6) a presents plots of time-dependence of the charge on each branch of the loop branched polymer for the case when sum rule in Eq.(3.1) is fulfilled. In Fig.(6) b similar plots are presented for the case, when sum rule in Eq. (3.1) is broken. For this case we solve sine-Gordon equation numerically. In Fig. ?? reflection coefficient of charged solitons from the polymer branching points are plotted for the regimes, when the sum rule given by Eq.(2.6) is fulfilled and broken. Again, the plot confirms absence of the reflection at the branching point in the regime, when the sum rule is fulfilled.

Another topology which can be exactly treated within the above approach is called tree-branching, which corresponds to hyperbranched conducting polymers. Such structure can be modeled in terms of metric tree graph presented in lower panel of Fig. 5. In simplest case, the graph consists of three "layers" $b_{1},\left(b_{1 i}\right),\left(b_{1 i j}\right)$, where $i, j$ run over the given branches. On each branch $b_{1}, b_{1 i}, b_{1 i j}$ we have a sine-Gordon equation given by (2.1). Writing on each branch of the graph Eq.(2.1), setting $\beta_{1}=\beta_{1 i}=$ $\beta_{1 i j}=1$ for all $i, j$, and assuming that the $a_{1 i}$ and $a_{1 i j}$ fulfill constraints in the form of sum rule, explicitly given, e.g. in [51], one can obtain exact soliton solutions of sine-
Gordon equation on the tree graph. Fig 8 a presents plots of the time-dependence of the charge on each branch of the tree-shaped conducting polymer, obtained using the exact solution of sine-Gordon equation on metric tree graph, i.e., for the case when sum rule for $b_{1}, b_{1 i}$ and $b_{1 i j}$ is fulfilled. Similar plots for the case when the sum rule is broken, is presented in Fig $8 \mathrm{~b}$. This latter clearly shows breaking of charge conservation law. We note that according to the conclusion made in [51] the above approach can be used for solving of sine-Gordon equation on metric graphs with arbitrary topology. The only requirement for the structure of such polymers is that it contains at least three very long, outgoing branches.

\section{CONCLUSIONS}

We studied charged soliton dynamics in branched conducting polymers. A model based on the sine-Gordon equation on metric graphs is proposed for the analysis of charged solitons transport in conducting polymers with different branching topologies. Time-dependence of the topological charge and kink scattering at the branching point are analyzed. The regime when there is no backstattering of charged solitons at the polymer branching points is revealed. Such regime corresponds to the case, when the sine-Gordon equation on metric graph is completely integrable and charge and energy conservation rules are fulfilled. For the polymers having many branching points, such regime implies ballistic charge transport. When the conservation rules are broken, transmission of charge carriers through the polymer branched point is accompanied by reflection. Such regime corresponds to diffusive transport of charged solitons through the polymer network. Existing the regime, when the transmission of charge carriers through the branching point is reflectionless makes possible to achieve very high conductivity in the polymer based organic electronic devices. The above model is applicable for arbitrary branching topology, provided the polymer consists of at least three very long branches and other branching part is located between these three branches. Finally, we note that our model allows to consider also breathers in branched polymers, using the breather solutions of sine-Gordon equation on metric graphs.
[1] R. J. Kline, M. D. McGehee, J. Macromolecular Sci. C, 4627 (2006).

[2] D. S. WaIlacet A. M. Stonehams, W. Hayest, A. J. Fishertt and A. Testas, J. Phys.: Condens. Matter 33905 (1991).

[3] D. Kumar, R. C. Sharma, Eur. Polym. J., 341053 (1998).

[4] W.R. Salaneck, R. H. Friend, J. L. BreHdas, Phys. Rep., 319231 (1999).

[5] A. J. Heeger and R. Pethig, Phil. Trans. R. Soc. Lond. A 31417 (1985).

[6] A. J. Heeger, Rev. Mod.Phys. 73681 (2001).
[7] A. J. Heeger, S. Kivelson, J. R. Schrieffer, W. -P. Su, Rev. Mod.Phys. 60781 (1988).

[8] D. S. WaIlace, A. M. Stoneham, W. Hayes, A. J. Fisher and A. Testa, J. Phys.: Condens. Matter, 3, 3905 (1991).

[9] M. Fujii, K. Ari and K. Yoshino, J. Electrochem. Soc., $1407,(1993)$.

[10] J. Jurkiewicz and A. Krzywicki Phys. Lett. B 392, 29 (1997).

[11] K. Inoue, Prog. Polym. Sci. 25, 453 (2000).

[12] L. Dai, B. Winkler, L. Dong, L. Tong and A. W. H. Mau, Adv. Mater., 13, 12-13 (2001). 
[13] D. T. Wu, Synth. Met., 126, 289 (2002).

[14] C. Gao and D. Yan Prog, Polym. Sci. 29, 183 (2004).

[15] F. Hua and E. Ruckenstein, Macromolecules 38, 888 (2005).

[16] R.-H. Lee, W.-Sh. Chen, and Y.-Y. Wang, Thin Solid Films, 517, 5747 (2009)

[17] A. Hirao and H.-S. Yoo, Polymer J., 43, 2 (2011).

18] L. R. Hutchings Macromolecules, 45, 5621 (2012).

[19] L. Pan,, G. Yu, D. Zhai, et.al, PNAS 109, 9287 (2012).

[20] J. Ohshita, Y. Tominaga, D. Tanaka, T. Mizumo, Y. Fujita, Y. Kunugi, J. Org. Chem., 50, 736 (2013).

[21] G. V. Otrokhov, O. V. Morozova, I. S. Vasileva, G. P. Shumakovich, E. A. Zaitseva, M. E. Khlupova, and A. I. Yaropolov, Biochemistry, 78, 1539 (2013).

[22] M. Goll, A. Ruff, E. Muks, F. Goerigk, B. Omiecienski, I. Ruff, R. C. Gonzalez-Cano, J. T. L. Navarrete, M. C. R. Delgado and S. Ludwigs, Beilstein J. Org. Chem., 11, 335 (2015).

[23] T. Soganci, O. Gumusay, H. C. Soyleyici, M. Ak, Polymer 134, 187 (2018).

[24] J. S. Andrade, Jr., N. Ito, Y. Shibusa, Phys. Rev. B, 54 3910 (1996).

[25] A. Fizazi, J. Moulton, K. Pakbaz, et.al., Phys. Rev. Lett., 642180 (1990).

[26] K. Maki, Synth. Met., 9185 (1984).

[27] L. Rothberg, T. M. Jedju, S. Etemad, G. L. Baker, Phys. Rev. Lett. 573229 (1982).

[28] M. Kuwabara, S. Abe, and Y. Ono, Synth. Met., 851109 (1997).

[29] P. B. Miranda, D. Moses, A. J. Heeger, Y. W. Park, Phys. Rev. B, 66125202 (2002).

[30] A. J. Heeger, S. Kivelson, J. R. Schrieffer, w. -p. Su, Rev. Mod.Phys. 60781 (1988).

[31] S. Brazovskii, Solid State Sci. 101786 (2008).

[32] S. Tretiak, A. Piryatinski, A. Saxena, R. L. Martin, A. R. Bishop, Phys. Rev. B, 70, 233203 (2004).
[33] W. P. Su, Schrieffer, A. J. Heeger, Phys. Rev. Lett., 42 1698 (1979).

[34] L. Bernasconi, J.Phys. Chem. Lett., 6908 (2015).

[35] M. Sasai, H. Fukutome, Prog. Theor. Phys., 7961 (1988).

[36] S. Suhai, J. Chem. Phys., 733843 (1980).

[37] V.Kostrykin and R.Schrader J. Phys. A: Math. Gen. 32 595 (1999)

[38] T.Kottos and U.Smilansky, Ann.Phys., 76274 (1999).

[39] P.Kuchment, Waves in Random Media, 14 S107 (2004).

[40] S.Gnutzmann and U.Smilansky, Adv.Phys. 55527 (2006).

[41] P.Exner and H.Kovarik, Quantum waveguides. (Springer, 2015).

[42] S.Gnutzmann, J.P.Keating, F. Piotet, Ann.Phys., 325 2595 (2010).

[43] Z.Sobirov, D.Matrasulov, K.Sabirov, S.Sawada, and K.Nakamura, Phys. Rev. E 81, 066602 (2010).

[44] Z. Sobirov, D. Matrasulov, S. Sawada, and K. Nakamura, Phys.Rev.E 84, 026609 (2011).

[45] R.Adami, C.Cacciapuoti, D.Finco, D.N., Rev.Math.Phys, 234 (2011).

[46] K.K.Sabirov, Z.A.Sobirov, D.Babajanov, and D.U.Matrasulov, Phys.Lett. A, 377, 860 (2013).

[47] J.-G.Caputo , D.Dutykh, Phys. Rev. E 90, 022912 (2014).

[48] H.Uecker, D.Grieser, Z.Sobirov, D.Babajanov and D.Matrasulov, Phys. Rev. E 91, 023209 (2015).

[49] D.Noja, Philos. Trans. R. Soc. A 372, 20130002 (2014).

[50] D.Noja, D.Pelinovsky, and G.Shaikhova, Nonlinearity 28, 2343 (2015).

[51] Z.Sobirov, D.Babajanov, D.Matrasulov, K.Nakamura, and H.Uecker, EPL 115, 50002 (2016).

[52] K.K.Sabirov, S. Rakhmanov, D. Matrasulov and H. Susanto Phys.Lett. A, 382, 1092 (2018). 